Document downloaded from:

http://hdl.handle.net/10251/40646

This paper must be cited as:

Bonet Solves, JA.; Vukoti, D. (2013). Superposition operators between weighted Banach spaces of analytic functions of controlled growth. Monatshefte für Mathematik. 170(34):311-323. doi:10.1007/s00605-012-0441-6.

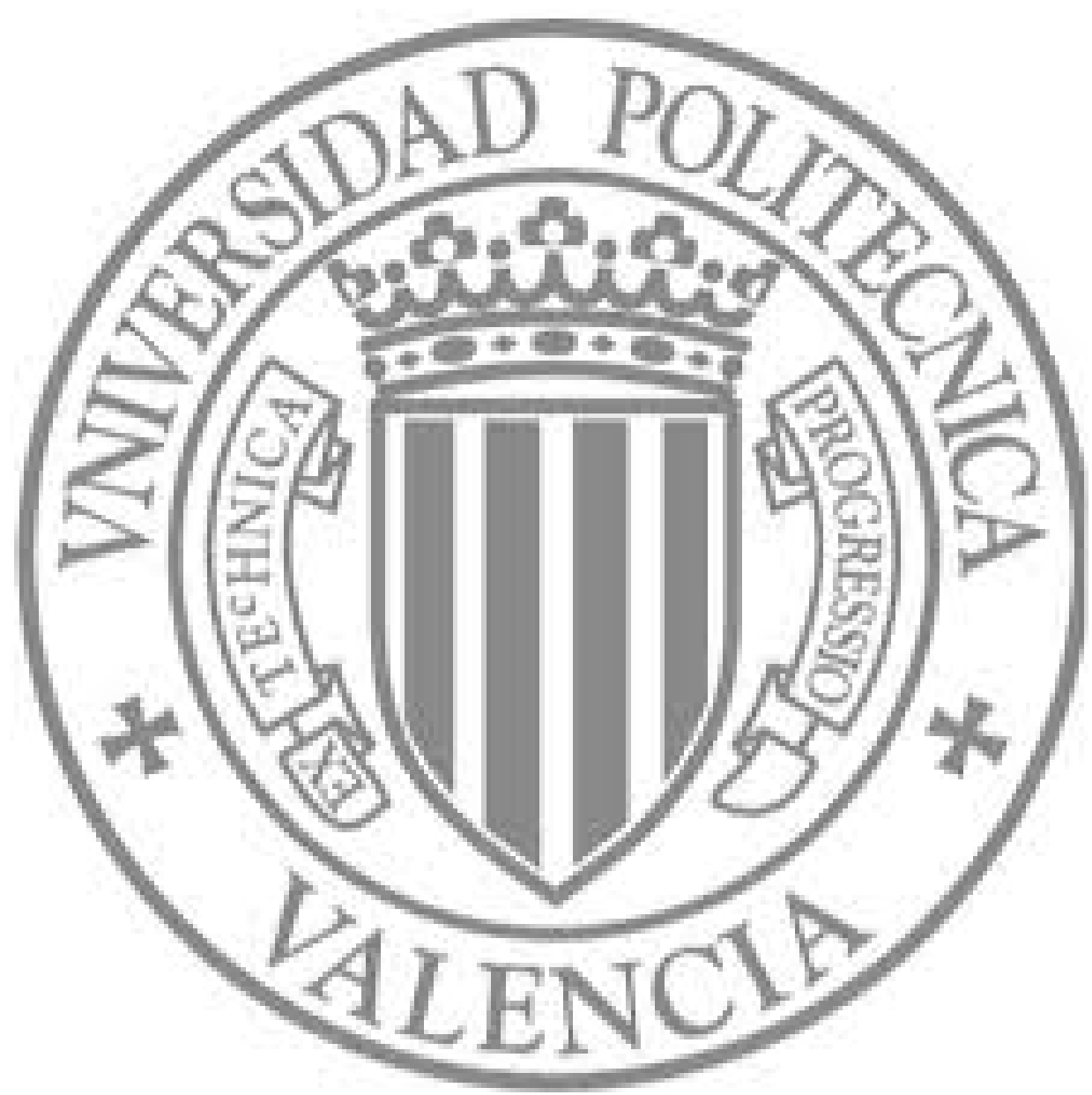

The final publication is available at

http://dx.doi.org/10.1007/s00605-012-0441-6

Copyright Springer Verlag (Germany) 


\title{
Superposition operators between weighted Banach spaces of analytic functions of controlled growth
}

\author{
José Bonet and Dragan Vukotić
}

\begin{abstract}
We characterize the entire functions which transform a weighted Banach space of holomorphic functions on the disc of type $H^{\infty}$ into another such space by superposition. We also show that all the superposition operators induced by such entire functions map bounded sets into bounded sets and are continuous. Superposition operators that map bounded sets into relatively compact sets are also considered.
\end{abstract}

\section{Authors' addresses:}

J. Bonet: Instituto Universitario de Matemática Pura y Aplicada IUMPA, Universitat Politècnica de València,

E-46022 Valencia, Spain

E-mail: jbonet@mat.upv.es

D. Vukotić: Departamento de Matemáticas

Universidad Autónoma de Madrid,

E-28049 Madrid, Spain

E-mail: dragan.vukotic@uam.es

2010 Mathematics Subject Classification. Primary: 47H30, Secondary: 46E15, 47B38

Key words and phrases. Superposition operators, weighted Banach spaces, entire functions. 


\section{Introduction and Notation}

The purpose of this note is to study superposition operators $f \rightarrow \varphi \circ f$ defined between weighted Banach spaces $H_{v}^{\infty}=H_{v}^{\infty}(\mathbb{D})$ of holomorphic functions on the disc by means of an entire function $\varphi$. If $X$ and $Y$ are linear spaces of holomorphic functions on the unit disc $\mathbb{D}$ of the complex plane and $\varphi$ is an entire function, the superposition operator $S_{\varphi}: X \rightarrow Y$ with symbol $\varphi$ is defined by $S_{\varphi}(f):=\varphi \circ f$. Since $X$ and $Y$ are assumed to be linear spaces, the operator $S_{\varphi}$ is linear if and only if $\varphi(z)=c z$ for some complex constant $c$ and all $z$. The central question concerning superposition operators is to characterize those symbols $\varphi$ such that the superposition operator maps $X$ into $Y$. In case $X$ and $Y$ are Banach spaces, it is also important to determine when $S_{\varphi}$ is bounded, in the sense that it maps bounded subsets of $X$ into bounded subsets of $Y$, when $S_{\varphi}$ is continuous or when it is compact, in the sense that it maps bounded sets into relatively compact sets.

Similar problems of action between spaces have a long history in the context of real valued functions $[2,3]$, where this operator is also known as the Nemytskij operator, and in the theory of uniform algebras. However, these questions in the context of complex functions have only been addressed during the last two decades or so. Superposition operators mapping a Bergman space or a Hardy space into another such space were characterized by Cámera and Giménez in [12] and Cámera [13]. A whole set of new techniques was used in obtaining a complete description of the superposition operators acting between various spaces of Dirichlet type in a paper by Buckley, Fernández and Vukotić [10]. Álvarez, Márquez, and Vukotić [1] described the superpositions between a Bergman space and the Bloch space in both directions in terms of the order and type of the entire function $\varphi$. More recently, Buckley and Vukotić [11] characterized superposition operators from an analytic Besov space into a Bergman space and Girela and Márquez [17] obtained analogous results for the superpositions between $Q_{p}$ and Hardy spaces. We also refer the reader to $[16],[28],[29]$, or to the survey [27].

The results in these papers are typically formulated in terms of a condition limiting the order and type of entire functions. In view of the first results of this type obtained in [10], the following question arose naturally around 2000: Can one find two natural scales of spaces $X_{\alpha}$ and $Y_{\beta}$ (depending of some exponents or indices $\alpha, \beta$ ) so that the characterization of all superposition operators $S_{\varphi}: X_{\alpha} \rightarrow Y_{\beta}$ would yield an alternative description of all entire functions of exponential type (covering the whole possible range of orders and types)? It is one of our aims in this article to give a partial answer to this question. See Proposition 3.2 and Theorem 3.4 for exact formulations.

In this paper a weight $v$ on $\mathbb{D}$ is a strictly positive continuous function on $\mathbb{D}$ which is radial, i.e. $v(z)=v(|z|), z \in \mathbb{D}, v(r)$ is strictly decreasing on $\left[0,1\left[\right.\right.$ and satisfies $\lim _{r \rightarrow 1} v(r)=0$. For such a weight, the weighted Banach space of holomorphic functions $H_{v}^{\infty}$ is defined by

$$
H_{v}^{\infty}:=\left\{f \in H(\mathbb{D})\left|\|f\|_{v}=\sup _{z \in \mathbb{D}} v(z)\right| f(z) \mid<+\infty\right\},
$$

endowed with the norm $\|f\|_{v}:=\sup _{z \in \mathbb{D}} v(z)|f(z)|$. Spaces of this type appear in the study of growth conditions of analytic functions and have been investigated in various articles since the work of Shields and Williams, see e.g. [4],[5], [22], [23], [26] and the references therein. Composition operators on these type of spaces have been also thoroughly studied [6], [7]. Harutyunyan and Lusky [19] investigated the continuity of the differentiation operator $D h=h^{\prime}$ between spaces of type $H_{v}^{\infty}$.

Here are examples of weights $v(z)$ on $\mathbb{D}$ satisfying our assumptions:

(1) The polynomial weights $v(z)=(1-|z|)^{\alpha}, \alpha>0$, which describe polynomial growth.

(2) The exponential weights $v(z)=\exp \left(-\frac{1}{(1-|z|)^{\alpha}}\right), \alpha>0$.

(3) The logarithmic weights $v(z)=\left(\log \frac{e}{1-|z|}\right)^{-\alpha}, \alpha>0$.

A few words about the notation. The greatest integer part of a real number $\gamma$ will be denoted by $[\gamma]$. For a given entire function $\varphi$, we will write $M(\varphi, r):=\sup _{|z|=r}|\varphi(z)|, r \geq 0$. We refer the reader to Levin [21] for the definition of type and order of an entire function. The Bloch space is denoted by $\mathcal{B}$. Among the many references on the Bloch functions, we mention [24] and [30]. 


\section{Some general results about boundedness and compact- ness}

Lemma 2.1 is a consequence of a more general result due to Boyd and Rueda [8, Theorem 1]. We present a short proof of the lemma in order to make the paper self-contained.

Lemma 2.1 Let $u$ and $v$ be weights. If the entire function $\varphi$ satisfies that the superposition operator $S_{\varphi}$ maps $H_{u}^{\infty}$ into $H_{v}^{\infty}$ and is bounded, then $S_{\varphi}: H_{u}^{\infty} \rightarrow H_{v}^{\infty}$ is continuous.

Proof. Since $S_{\varphi}: H_{u}^{\infty} \rightarrow H_{v}^{\infty}$ is bounded, it is locally bounded in the sense that it is bounded on a neighbourhood of each point ( $c f$. Dineen [14, p. 10]). By [14, Lemma 2.8], to show that $S_{\varphi}: H_{u}^{\infty} \rightarrow H_{v}^{\infty}$ is continuous, it is enough to show that, for each $f, g \in H_{u}^{\infty}$, the map $\Phi: \lambda \in$ $\mathbb{C} \rightarrow S_{\varphi}(g+\lambda f)$ is holomorphic. As $\Phi$ is also locally bounded and the linear span of the Dirac functionals $\delta_{\zeta}, \zeta \in \mathbb{D}$, is weak-* dense in $\left(H_{v}^{\infty}\right)^{*}$, by a result of Grosse-Erdmann [18, Theorem 1], $\Phi$ is holomorphic if we show that, for each $\zeta \in \mathbb{D}$, the map

$$
\lambda \in \mathbb{C} \mapsto\left(\delta_{\zeta} \circ \Phi\right)(\lambda)=\varphi(g(\zeta)+\lambda f(\zeta))
$$

is holomorphic. However, this is trivial because $\varphi$ is an entire function.

We are now ready for a general result.

Theorem 2.2 (a) Let $u$ and $v$ be weights. If the entire function $\varphi$ satisfies the following condition:

$$
\begin{gathered}
\forall \varepsilon \in] 0,1\left[\exists C>0 \exists R_{0}>0 \forall R \geq R_{0}:\right. \\
v\left(u^{-1}\left(\frac{1}{\varepsilon R}\right)\right) \max _{|w|=R}|\varphi(w)| \leq C,
\end{gathered}
$$

then the superposition operator $S_{\varphi}$ maps $H_{u}^{\infty}$ into $H_{v}^{\infty}$ and is bounded.

(b) Let $u$ and $v$ be weights. If the entire function $\varphi$ satisfies the following condition:

$$
\lim _{R \rightarrow \infty} v\left(u^{-1}\left(\frac{k}{R}\right)\right) M(\varphi, R)=0
$$

for each $k \in \mathbb{N}$, then $S_{\varphi}: H_{u}^{\infty} \rightarrow H_{v}^{\infty}$ is compact.

Proof. (a) Fix $f \in H_{u}^{\infty}$ with $\|f\|_{u} \leq M, M>1$. Put $\varepsilon:=1 / M$, find $C, R_{0}$ as in the assumption and set

$$
A:=\max \left(R_{0}, M / u(0)\right), B:=\max _{|w| \leq A}|\varphi(w)| .
$$

If $|f(z)| \leq A$, then $v(z)|\varphi(f(z))| \leq v(0) B$. If $|f(z)|>A$, since $u$ and $u^{-1}$ are strictly decreasing, we have that $u^{-1}\left(1 /(\varepsilon|f(z)|)\right.$ is defined and $|z| \geq u^{-1}(1 /(\varepsilon|f(z)|)$. Applying the fact that $v$ is decreasing, we get $v(z) \leq v\left(u^{-1}(1 /(\varepsilon|f(z)|))\right)$. This implies $v(z)|\varphi(f(z))| \leq C$. Consequently

$$
\|\varphi \circ f\|_{v} \leq \max (v(0) B, C),
$$

and $S_{\varphi}\left(H_{u}^{\infty}\right) \subset H_{v}^{\infty}$ and $S_{\varphi}$ is bounded.

(b) For each $k \in \mathbb{N}$ and $0 \leq r<1$, define $\psi_{k}(r):=M(\varphi, k / u(r))$. Clearly $\psi_{k}$ is strictly increasing, $\psi_{k}(r) \rightarrow \infty$ as $r \rightarrow 1, \psi_{k}(0)=M(\varphi, k / u(0))$, and $\psi_{k} \leq \psi_{k+1}$ on $[0,1[$ for each $k \in \mathbb{N}$. We show that $\psi_{k}(r)=o(1 / v(r))$ as $r \rightarrow 1$ for all $k \in \mathbb{N}$. Indeed, fix $k \in \mathbb{N}$ and $\delta>0$. Apply the hypothesis to find $R_{0}>0$ such that $v\left(u^{-1}\left(\frac{k}{R}\right)\right) M(\varphi, R)<\delta$ if $R \geq R_{0}$. We assume that $R_{0}$ is selected so that $k / R_{0}<u(0)$, so that $u^{-1}(k / R)$ is defined if $R \geq R_{0}$. Now, if $u^{-1}\left(k / R_{0}\right)<r<1$, then $k / u(r)>R_{0}$. Therefore

$$
v\left(u^{-1}\left(\frac{k}{k / u(r)}\right)\right) M(\varphi, k / u(r))=v(r) M(\varphi, k / u(r))=v(r) \psi_{k}(r)<\delta .
$$


Now we construct $\psi:\left[0,1[\rightarrow] o, \infty\left[\right.\right.$, strictly increasing, continuous such that $\psi_{k}(r)=o(\psi(r))$ as $r \rightarrow 1$ for each $k \in \mathbb{N}$ and $\psi(r)=o(1 / v(r))$ as $r \rightarrow 1$. Clearly $w(z):=1 / \psi(z), z \in \mathbb{D}$, is a weight and $\lim _{r \rightarrow 1} v(r) / w(r)=0$. This implies that the inclusion $H_{w}^{\infty} \subset H_{v}^{\infty}$ is compact as a consequence of Montel's theorem. See also [7] for more general results. The proof is complete if we show that $S_{\varphi}: H_{u}^{\infty} \rightarrow H_{w}^{\infty}$ is continuous. To see this we apply part (a) and Lemma 2.1. Fix $\varepsilon>0$ and select $k \in \mathbb{N}$ with $k>1 / \varepsilon$. Since $\psi_{k}(r)=o(1 / w(r))$ as $r \rightarrow 1$, there is $\left.r_{0} \in\right] 0,1[$ such that $M(\varphi, k / u(r)) \leq 1 / w(r)$. Set $R_{0}:=k / u\left(r_{0}\right)$. For $R \geq R_{0}$ we have $0<k / R \leq k / R_{0}=u\left(r_{0}\right)<u(0)$, hence $u^{-1}(k / R)$ is defined and $u^{-1}(k / R) \geq r_{0}$, since $u$ is strictly decreasing. Thus

$$
M(\varphi, R) w\left(u^{-1}(1 / \varepsilon R)\right) \leq M(\varphi, R) w\left(u^{-1}(k / R)\right)=M\left(\varphi, k / u\left(u^{-1}(k / R)\right)\right) w\left(u^{-1}(k / R)\right) \leq 1,
$$

which proves the sufficient condition in part (a).

\section{Results for some concrete weights}

\subsection{The domain space is defined by a polynomial weight}

Below we will use the notation $[\gamma]$, as is customary, to denote the greatest integer part of a real number $\gamma$.

Proposition 3.1 Let $u(z)=(1-|z|)^{\alpha}, \alpha>0, v(z)=(1-|z|)^{\beta}, \beta>0$.

(1) The following conditions are equivalent for an entire function $\varphi$ :

(i) $\varphi$ is a polynomial of degree at most $[\beta / \alpha]$.

(ii) The superposition operator $S_{\varphi}$ maps $H_{u}^{\infty}$ into $H_{v}^{\infty}$.

(iii) The superposition operator $S_{\varphi}$ maps $H_{u}^{\infty}$ into $H_{v}^{\infty}$ and is bounded.

(2) The following conditions are equivalent for an entire function $\varphi$ :

(i) $\varphi$ is a polynomial of degree $s<\beta / \alpha$.

(ii) The superposition operator $S_{\varphi}$ maps $H_{u}^{\infty}$ into $H_{v}^{\infty}$ and it is compact, i.e. maps bounded sets into relatively compact sets.

Proof. We first prove the equivalences in part (1).

Condition (i) implies condition (iii) by Theorem 2.2 (a) since $v\left(u^{-1}(w)\right)=|w|^{\beta / \alpha}$. Clearly condition (iii) implies condition (ii).

Now assume that (ii) holds and set $m:=[\beta / \alpha]$. To conclude (i), by the standard Cauchy estimates, it is enough to prove $\lim _{r \rightarrow \infty} M(\varphi, r) / r^{m+1}=0$. Assuming that this does not hold, we select a sequence $\left(w_{n}\right)_{n}$ of complex numbers such that $\left|w_{n}\right|>1$ for each $n \in \mathbb{N}, \lim _{n \rightarrow \infty}\left|w_{n}\right|=\infty$ and $\left|\varphi\left(w_{n}\right)\right|>\delta\left|w_{n}\right|^{m+1}$ for each $n \in \mathbb{N}$ and some $\delta>0$. By passing to a subsequence, we may assume without loss of generality that all the elements in the sequence $\left(w_{n}\right)_{n}$ also satisfy $\left|\arg w_{n}\right|<$ $\frac{1}{4} \pi \alpha$. This can be assumed safely after applying symmetries and rotations if necessary, since the entire functions $\psi(z):=\overline{\varphi(\bar{z})}$ and $\varphi_{t}(z):=\varphi\left(e^{i t} z\right)$ behave like $\varphi$ with respect to condition (i). The function $g(z)=(1-z)^{-\alpha}$ belongs to $H_{u}^{\infty}$. The preimages of $w_{n}$ under $g$ :

$$
z_{n}=1-\frac{1}{w_{n}^{1 / \alpha}}, \quad\left(1-z_{n}\right)^{-1}=w_{n}^{1 / \alpha},
$$

satisfy $\left|1-z_{n}\right|<1$ and $\left|\arg \left(1-z_{n}\right)\right|<\pi / 4$; i.e. they belong to a Stolz domain, hence there is $c>0$ such that $\left|1-z_{n}\right| \leq c\left(1-\left|z_{n}\right|\right)$ for each $n \in \mathbb{N}$.

Suppose that $S_{\varphi} g=\varphi \circ g$ belongs to $H_{v}^{\infty}$. There is $M>0$ such that $|\varphi(g(z))| \leq M(1-|z|)^{-\beta}$ for all $z \in \mathbb{D}$. This implies

$$
\delta\left|w_{n}\right|^{m+1}<\left|\varphi\left(w_{n}\right)\right|=\left|\varphi\left(g\left(z_{n}\right)\right)\right| \leq M\left(1-\left|z_{n}\right|\right)^{-\beta} \leq M c^{\beta}\left|1-z_{n}\right|^{-\beta}=M c^{\beta}\left|w_{n}\right|^{\beta / \alpha} .
$$


Consequently $\left|w_{n}\right|^{m+1-\beta / \alpha} \leq M c^{\beta} \delta^{-1}$ for each $n \in \mathbb{N}$, which is a contradiction, since $m+1-\beta / \alpha$ is strictly positive.

Now we prove (2). Assume first that (i) holds and denote by $s$ the degree of the polynomial $\varphi$. By assumption $s \alpha<\beta$. For each $k \in \mathbb{N}$, we have

$$
v\left(u^{-1}(k / R)\right) M(\varphi, R)=(k / R)^{\beta / \alpha} M(\varphi, R)=k^{\beta / \alpha} \frac{M(\varphi, R)}{R^{\beta / \alpha}},
$$

which tends to 0 as $R \rightarrow \infty$. We can apply Theorem 2.2 (b) to conclude that $S_{\varphi}$ maps $H_{u}^{\infty}$ into $H_{v}^{\infty}$ and is compact. Conversely, if $S_{\varphi}$ maps $H_{u}^{\infty}$ into $H_{v}^{\infty}$ and it is compact, then we conclude from part (1) that $s \leq \beta / \alpha$.

It remains to show that if $\beta=s \alpha$, then $S_{\varphi}: H_{u}^{\infty} \rightarrow H_{u^{s}}^{\infty}$ is not compact, $u^{s}(z)=u(z)^{s}=$ $(1-|z|)^{\beta}$. We show this for $\varphi(w)=w^{s}$, which is enough. Consider a strictly increasing sequence of positive numbers $\left(r_{n}\right)_{n}$ tending to 1 and define $g_{n}(z):=\left(1-r_{n}\right)^{\alpha} /\left(1-r_{n} z\right)^{2 \alpha}, z \in \mathbb{D}$. Clearly $g_{n} \in H_{u}^{\infty},\left\|g_{n}\right\|_{u} \leq 1$ for each $n \in \mathbb{N}$ and the sequence $\left(g_{n}\right)_{n}$ tends to 0 uniformly on compact sets. As $\beta=s \alpha, S_{\varphi}\left(g_{n}\right)(z)=\left(1-r_{n}\right)^{\beta} /\left(1-r_{n} z\right)^{2 \beta}, z \in \mathbb{D}$, and $S_{\varphi}\left(g_{n}\right)$ converges to 0 uniformly on compact sets. If $S_{\varphi}: H_{u}^{\infty} \rightarrow H_{u^{s}}^{\infty}$ were compact, the sequence $S_{\varphi}\left(g_{n}\right)$ would converge to 0 in $H_{u^{s}}^{\infty}$. This is a contradiction since $\left(1-r_{n}\right)^{\beta} S_{\varphi}\left(g_{n}\right)\left(r_{n}\right)=1 /\left(1+r_{n}\right)^{2 \beta}$ converges to $1 / 4^{\beta}$ as $n$ goes to infinity.

Observe that every entire function $\varphi$ defines a bounded superposition operator $S_{\varphi}$ from the space $H^{\infty}$ of bounded analytic functions on $\mathbb{D}$ into an arbitrary weighted Banach space of type $H_{v}^{\infty}$, since $\varphi \circ f \in H^{\infty} \subset H_{v}^{\infty}$ for each $f \in H^{\infty}$. The space $H^{\infty}$ corresponds to the "weight" $v(z)=1, z \in \mathbb{D}$, which is not a weight in the sense of this paper. On the other hand, the only superposition operators $S_{\varphi}$ mapping $H_{u}^{\infty}$ into $H_{v}^{\infty}$ for $u(z)=(1-|z|)^{2}, v(z)=1-|z|, z \in \mathbb{D}$, are those with $\varphi$ constant.

Proposition 3.2 (1) was obtained independently by Boyd and Rueda [9, Theorem 3].

Proposition 3.2 Let $u(z)=(1-|z|)^{\alpha}, z \in \mathbb{D}$ and $v(z)=\exp \left(-\frac{1}{(1-|z|)^{\beta}}\right), \alpha, \beta>0$. Let $\varphi$ be an entire function.

(1) If the function $\varphi$ is of order less than $\beta / \alpha$ or of order $\beta / \alpha$ and type zero, or equivalently, if for all $0<\varepsilon<1$ there are $C \geq 1, R_{0}>0$ such that $|\varphi(z)| \leq C \exp \left(\varepsilon|z|^{\beta / \alpha}\right)$ for all $z \in \mathbb{C}$ with $|z| \geq R_{0}$, then the superposition operator $S_{\varphi}$ maps $H_{u}^{\infty}$ into $\bar{H}_{v}^{\infty}$ and is bounded.

(2) If the superposition operator $S_{\varphi}$ maps $H_{u}^{\infty}$ into $H_{v}^{\infty}$, then for each $D>1$ we can find $C \geq$ $1, R_{0}>0$ such that $|\varphi(z)| \leq C \exp \left(D|z|^{\beta / \alpha}\right)$ for all $z \in \mathbb{C}$ with $|z| \geq R_{0}$.

Proof. Part (1) follows from Theorem $2.2(\mathrm{a})$, since $v\left(u^{-1}(s)\right)=\exp \left(-1 / s^{\beta / \alpha}\right)$. To prove (2), we proceed by reduction to absurd. If the desired conclusion does not hold, we can find $D>1$ such that for all $n \in \mathbb{N}$ there is $w_{n} \in \mathbb{C},\left|w_{n}\right|>n$, such that $\left|\varphi\left(w_{n}\right)\right| \geq n \exp \left(D\left|w_{n}\right|^{\beta / \alpha}\right)$. Given $D$, select $d>1$ with $1<d^{\beta}<D$ and find $0<\theta<\frac{\pi \alpha}{4}$ such that, if $z$ belongs to the Stolz angle

$$
S_{\theta}:=\{z \in \mathbb{D}|| 1-z|<1,| \arg (1-z) \mid<\theta\}
$$

then $|1-z| \leq d(1-|z|)$. Passing to a subsequence, using rotations and symmetries as in the proof of Proposition 3.1, we may assume that all $w_{n}$ belong to the first quadrant, the sequence $\left(\arg w_{n}\right)_{n}$ converges to 0 and $\arg w_{n} \leq \beta \theta$. This is possible because the entire functions $\psi(z):=\overline{\varphi(\bar{z}))}$ and $\varphi_{t}(z):=\varphi\left(e^{i t} z\right)$ behave like $\varphi$ with respect to condition (i). The function $g(z)=(1-z)^{-\alpha}$ belongs to $H_{u}^{\infty}$. By condition (ii), $\varphi \circ g \in H_{v}^{\infty}$. The preimages of $w_{n}$ under $g$

$$
z_{n}=1-\frac{1}{w_{n}^{1 / \alpha}},\left(1-z_{n}\right)^{-\beta}=w_{n}^{\beta / \alpha},
$$

belong to the Stolz domain $S_{\theta}$. In particular $\left|1-z_{n}\right| \leq d\left(1-\left|z_{n}\right|\right)$ for each $n \in \mathbb{N}$. We have, for each $n \in \mathbb{N}$,

$$
\left|\varphi\left(g\left(z_{n}\right)\right)\right|=\left|\varphi\left(w_{n}\right)\right|>n \exp \left(D\left|w_{n}\right|^{\beta / \alpha}\right)=
$$




$$
=n \exp \left(\frac{D}{\left|1-z_{n}\right|^{\beta}}\right) \geq n \exp \left(\frac{D}{d^{\beta}\left(1-\left|z_{n}\right|\right)^{\beta}}\right) \geq n \exp \left(\frac{1}{\left(1-\left|z_{n}\right|\right)^{\beta}}\right) .
$$

This contradicts $\varphi \circ g \in H_{v}^{\infty}$ and completes the proof.

\subsection{The domain space is defined by a logarithmic weight}

The key result to treat the case of logarithmic weights in the domain is the following Lemma. It is based on a geometric construction of a simply connected domain that can be imagined as a "highway from a point near the origin to infinity" of constant width. It is a variant of the construction to be found e.g. in [1, Lemma 2], [11, Lemma 7].

Lemma 3.3 For each positive number $\delta$ and for every sequence $\left\{w_{n}\right\}_{n=0}^{\infty}$ of complex numbers such that $\left|w_{0}\right|<\delta,\left|w_{1}\right| \geq 3 \delta, 0<\arg w_{1}<\pi / 4, \arg w_{n} \searrow 0$, and

$$
\left|w_{n}\right| \geq \max \left\{3\left|w_{n-1}\right|, 2 \sum_{k=1}^{n-1}\left|w_{k}-w_{k-1}\right|\right\} \quad \text { for all } n \geq 2,
$$

there exists a domain $\Omega$ with the following properties:

(i) $\Omega$ is simply connected;

(ii) $\Omega$ contains the infinite polygonal line $L=\cup_{n=1}^{\infty}\left[w_{n-1}, w_{n}\right]$, where $\left[w_{n-1}, w_{n}\right]$ denotes the line segment from $w_{n-1}$ to $w_{n}$;

(iii) any Riemann map $f$ of $\mathbb{D}$ onto $\Omega$ belongs to the Bloch space $\mathcal{B}$;

(iv) $\operatorname{dist}(w, \partial \Omega)=\delta$ for each point $w$ on the broken line $L$.

Proof. It is clear from (3.1) that $\left|w_{n}\right| \nearrow \infty$ as $n \rightarrow \infty$. We construct the domain $\Omega$ as follows. First connect the points $w_{n}$ by a polygonal line $L$ as indicated in the statement. Let $D(z, \delta)=$ $\{w:|z-w|<\delta\}$ and define

$$
\Omega=\bigcup\{D(z, \delta): z \in L\},
$$

i.e. let $\Omega$ be a $\delta$-thickening of the polygonal line $L$. In other words, $\Omega$ is the union of simply connected cigar-shaped domains

$$
C_{n}=\bigcup\left\{D(z, \delta): z \in\left[w_{n-1}, w_{n}\right]\right\} .
$$

By our choice of $w_{n}$, it is easy to check inductively that $\left|w_{n}-w_{k}\right| \geq 5 \delta$ whenever $n>k$. Since our construction implies that

$$
C_{n} \subset\left\{w:\left|w_{n-1}\right|-\delta<|w|<\left|w_{n}\right|+\delta\right\},
$$

wee see immediately that

(a) for all $m, n, C_{m} \cap C_{n} \neq \emptyset$ if and only if $|m-n| \leq 1$;

(b) for all $n, C_{n} \cap C_{n+1}$ is either $D\left(w_{n}, \delta\right)$ or the interior of the convex hull of $D\left(w_{n}, \delta\right) \cup\left\{a_{n}\right\}$ for some point $a_{n}$ outside of $\overline{D\left(w_{n}, \delta\right)}$.

Thus, each $\Omega_{N}=\cup_{n=1}^{N} C_{n}$ is also simply connected. Since

$$
\Omega=\cup_{N=1}^{\infty} \Omega_{N} \quad \text { and } \quad \Omega_{N} \subset \Omega_{N+1} \text { for all } N,
$$

we conclude that $\Omega$ is also simply connected by the Cauchy integral theorem and a simple compactness argument (open coverings). By construction, $\operatorname{dist}(w, \partial \Omega) \leq \delta$ for all $w$ in $\Omega$, hence any Riemann map onto $\Omega$ will belong to $\mathcal{B}$. It is also clear that (iv) holds. 
Recall that the hyperbolic distance between two points $z$ and $w$ in the disk is defined as

$$
\rho(z, w)=\inf _{\gamma} \int_{\gamma} \frac{|d \zeta|}{1-|\zeta|^{2}}=\frac{1}{2} \log \frac{1+\left|\frac{z-w}{1-\bar{z} w}\right|}{1-\left|\frac{z-w}{1-\bar{z} w}\right|},
$$

where the infimum is taken over all rectifiable curves $\gamma$ in $\mathbb{D}$ that join $z$ with $w$.

The hyperbolic metric $\rho_{\Omega}$ on an arbitrary simply connected domain $\Omega$ (not the entire plane) is defined via the corresponding pullback to the disk: if $f$ is a Riemann map of $\mathbb{D}$ onto $\Omega$ then

$$
\rho_{\Omega}(f(z), f(w))=\rho(z, w)=\inf _{\Gamma} \int_{f^{-1}(\Gamma)} \frac{|d \zeta|}{1-|\zeta|^{2}},
$$

where the infimum is taken over all rectifiable curves $\Gamma$ in $\Omega$ from $f(z)$ to $f(w)$. The metric $\rho_{\Omega}$ does not depend on the choice of the Riemann map $f$. For more details, we refer the reader to $\S 1.2$ and $\S 4.6$ of [24].

From the definition of hyperbolic metric we notice the following important feature of Riemann maps:

$$
\rho_{\Omega}(f(0), f(z))=\rho(0, z) \geq \frac{1}{2} \log \frac{1}{1-|z|}, \quad z \in \mathbb{D} .
$$

Another fundamental property is the comparison between the hyperbolic and the so-called quasihyperbolic metric:

$$
\rho_{\Omega}\left(w_{1}, w_{2}\right) \leq \inf _{\Gamma} \int_{\Gamma} \frac{|d w|}{\operatorname{dist}(w, \partial \Omega)},
$$

where the infimum is taken over all rectifiable curves $\Gamma$ in $\Omega$ from $w_{1}$ to $w_{2}$.

Theorem 3.4 Let $u(r)=\left(\log \frac{e}{1-r}\right)^{-\alpha}$ and $v(r)=(1-r)^{\beta}$ with $\alpha, \beta>0$. The following statements are equivalent for an entire function $\varphi$ :

(i) The function $\varphi$ is of order less than $1 / \alpha$ or of order $1 / \alpha$ and type zero.

(ii) For all $0<\varepsilon<1$ there are $C>0, R_{0}>0$ such that $|\varphi(z)| \leq C \exp \left(\varepsilon|z|^{1 / \alpha}\right)$ whenever $|z| \geq R_{0}$

(iii) The superposition operator $S_{\varphi}$ maps $H_{u}^{\infty}$ into $H_{v}^{\infty}$

(iv) $S_{\varphi}$ is a bounded operator from $H_{u}^{\infty}$ into $H_{v}^{\infty}$.

(v) $S_{\varphi}$ is a compact operator from $H_{u}^{\infty}$ into $H_{v}^{\infty}$.

Proof. Conditions (i) and (ii) are equivalent by the definition of type and order. We show that $(\mathrm{ii}) \Rightarrow(\mathrm{v})$. A direct calculation shows that $v\left(u^{-1}(s)\right)=e^{\beta} \exp \left(-\beta / s^{1 / \alpha}\right)$. In order to apply Theorem 2.2 (b) to conclude that $S_{\varphi}: H_{u}^{\infty} \rightarrow H_{v}^{\infty}$ is compact, we fix $k \in \mathbb{N}$ and select $\varepsilon>0$ such that $\varepsilon<\beta / k^{1 / \alpha}$. We apply condition (ii) to find $C>0$ and $R_{0}>0$. If $R \geq R_{0}$, we have

$$
v\left(u^{-1}(k / R)\right) M(\varphi, R)=e^{\beta} \exp \left(-\frac{\beta R^{1 / \alpha}}{k^{1 / \alpha}}\right) M(\varphi, R) \leq C e^{\beta} \exp \left(\left(-\frac{\beta}{k^{1 / \alpha}}+\varepsilon\right) R^{1 / \alpha}\right),
$$

that tends to 0 as $R \rightarrow \infty$.

For the proof of (iii) $\Rightarrow\left(\right.$ ii), let us assume that $S_{\varphi}\left(H_{u}^{\infty}\right) \subset H_{v}^{\infty}$ holds but (ii) is false. Then we can find $\varepsilon>0$ and a sequence $\left(w_{n}\right)_{n}^{\infty}$ such that

$$
\left|\varphi\left(w_{n}\right)\right| \geq n e^{\varepsilon\left|w_{n}\right|^{1 / \alpha}}, \quad \text { for all } n .
$$

Select a positive constant $\delta>\beta / \varepsilon$. By passing on to a subsequence, denoted again $\left(w_{n}\right)_{n}$, we may assume that the sequence $\left(\arg w_{n}\right)_{n}$ in $[0,2 \pi]$ is convergent and all points $w_{n}$ lie in an angular sector of opening $\left\{w:|\arg w|<\frac{\pi}{4}\right\}$. We can further assume that they are all located in 
the first quadrant and the arguments $\arg w_{n}$ decrease to 0 , by applying symmetries or rotations if necessary. There is no loss of generality in doing this because the entire functions $\psi, \varphi_{t}$, defined by $\psi(z)=\overline{\varphi(\bar{z})}, \varphi_{t}(z)=\varphi\left(e^{i t} z\right)$ respectively, behave like $\varphi$ with respect to condition (ii).

Select inductively a further subsequence, labelled again $\left(w_{n}\right)_{n}$, so that $\left|w_{1}\right| \geq 3 \delta$, and inequality (3.1) holds. Next, add to the sequence a point $w_{0} \neq 0$ with $\left|w_{0}\right|<\delta$ and use Lemma 3.3 to construct a domain $\Omega$ with the properties (i)-(iv) indicated there, contained in the angular sector $\left\{w:|\arg w|<\frac{\pi}{4}\right\}$. Let $f$ be a Riemann map of $\mathbb{D}$ onto $\Omega$ such that $f(0)=w_{0}$.

Now let $z_{n}$ be the pre-images in $\mathbb{D}$ of the points $w_{n}, n \geq 1$. Since $\left|w_{n}\right| \rightarrow \infty$ as $n \rightarrow \infty$, it follows that $\left|z_{n}\right| \rightarrow 1$. By applying estimate (3.2) for hyperbolic metric, the triangle inequality, inequality (3.3) and property (iv) from Lemma 3.3, as well as the property (3.1) of the points $w_{n}$ respectively, we obtain the following chain of inequalities:

$$
\begin{aligned}
\frac{1}{2} \log \frac{1}{1-\left|z_{n}\right|} & \leq \rho_{\Omega}\left(f(0), f\left(z_{n}\right)\right) \leq \sum_{k=1}^{n} \rho_{\Omega}\left(w_{k-1}, w_{k}\right) \leq \sum_{k=1}^{n} \int_{\left[w_{k-1}, w_{k}\right]} \frac{|d w|}{\operatorname{dist~}(w, \partial \Omega)} \\
& =\sum_{k=1}^{n} \int_{\left[w_{k-1}, w_{k}\right]} \frac{|d w|}{\delta}=\frac{1}{\delta} \sum_{k=1}^{n}\left|w_{k}-w_{k-1}\right| \leq \frac{1}{2 \delta}\left|w_{n}\right| .
\end{aligned}
$$

This shows that

$$
\left|w_{n}\right| \geq \delta \log \frac{1}{1-\left|z_{n}\right|}, \quad n \geq 1 .
$$

Since $\Omega$ is a simply connected domain that does not contain the origin and the function $f$ does not vanish in the disk, we can define the analytic function $F=f^{\alpha}$. As observed, the function $f \in \mathcal{B}$, so by the basic logarithmic estimate for Bloch functions, the function $F$ enjoys the estimate

$$
|F(z)| \leq K\left(\log \frac{1}{1-|z|}\right)^{\alpha}, \quad z \in \mathbb{D},
$$

hence $F \in H_{u}^{\infty}$.

On the other hand, by (3.5), we also know that

$$
\left|F\left(z_{n}\right)\right|=\left|w_{n}\right|^{\alpha} \geq \delta^{\alpha}\left(\log \frac{1}{1-\left|z_{n}\right|}\right)^{\alpha}, \quad z \in \mathbb{D} .
$$

From here and our assumption on $\varphi$ and $w_{n}$ we deduce that

$$
\left|\varphi\left(F\left(z_{n}\right)\right)\right| \geq n e^{\varepsilon\left|F\left(z_{n}\right)\right|^{1 / \alpha}} \geq n e^{\varepsilon \delta \log \frac{1}{1-\left|z_{n}\right|}} \geq \frac{n}{\left(1-\left|z_{n}\right|\right)^{\beta}},
$$

which means that $\varphi \circ F \notin H_{v}^{\infty}$. This contradicts our assumption $S_{\varphi}: H_{u}^{\infty} \rightarrow H_{v}^{\infty}$, which completes the proof.

By proceeding similarly as in the proof of Proposition 3.1, and using Lemma 3.3, we can prove the following result.

Proposition 3.5 Let $u(r)=\left(\log \frac{e}{1-r}\right)^{\alpha}, \alpha>0, v(r)=\left(\log \frac{e}{1-r}\right)^{\beta}, \beta>0$.

(1) The superposition operator $S_{\varphi}$ maps $H_{u}^{\infty}$ into $H_{v}^{\infty}$ and is bounded if and only if $\varphi$ is a polynomial of degree at most $[\beta / \alpha]$.

(2) The superposition operator $S_{\varphi}$ maps $H_{u}^{\infty}$ into $H_{v}^{\infty}$ and is compact if and only if $\varphi$ is a polynomial of degree less than $\beta / \alpha$.

As a closing remark, we would like to stress that if one is concerned only with the boundedness of superposition operators, most of the statements of this section can be proved in a different way. For example, proving that the statements (i), (ii), and (iv) in Theorem 3.4 are equivalent no longer requires an involved geometric constructions. Relatively general results of this type were obtained most recently by Ramos Fernández [25] for some known classes of weights such as the so-called essential weights [7]. 


\subsection{Superpositions from a space defined by an exponential weight}

Proposition 3.6 Let $u(r)=\exp \left(-(1-|z|)^{-\alpha}\right), \alpha>0$ and let $\varphi$ be an entire function.

(1) If there exist $C>0$ and $R_{0}>0$ such that $|\varphi(w)| \leq C \exp \left((\log |w|)^{\gamma}\right)$ for $|w| \geq R_{0}$, then for each $c>1$ the superposition operator $S_{\varphi}$ maps $H_{u}^{\infty}$ boundedly into the space $H_{v_{c}}^{\infty}$, where $v_{c}(r)=\exp \left(-\frac{c}{(1-|z|)^{\alpha \gamma}}\right)$.

(2) If the superposition operator $S_{\varphi}$ maps $H_{u}^{\infty}$ into $H_{v}^{\infty}, v(r)=\exp \left(-\frac{1}{(1-|z|)^{\beta}}\right), \beta>0$, then for every $c>1$ there exist $C>0$ and $R_{0}>0$ such that $|\varphi(w)| \leq C \exp \left(c(\log |w|)^{\beta / \alpha}\right)$ for $|w| \geq R_{0}$.

Proof. We first prove (1). Fix $c>1$. Given $f \in H_{u}^{\infty}$, there is a constant $M>0$ such that

$$
\log |f(z)| \leq \log M+(1-|z|)^{-\alpha} .
$$

Select $\left.r_{0} \in\right] 0,1\left[\right.$ so that $\log M \leq\left(c^{1 / \gamma}-1\right) /(1-|z|)^{\alpha}$ for $r_{0} \leq|z|<1$. If $|f(z)| \geq R_{0}$ and $r_{0} \leq|z|<1$ we have

$$
|\varphi(f(z))| \leq C \exp \left((\log |f(z)|)^{\gamma}\right) \leq C \exp \left(\left(\log M+1 /(1-|z|)^{\alpha}\right)^{\gamma}\right) \leq C \exp \left(\frac{c}{(1-|z|)^{\alpha \gamma}}\right) .
$$

On the other hand, there is $S_{0} \geq R_{0}$ such that $|f(z)| \leq S_{0}$ if $|z| \leq r_{0}$. Hence $|\varphi(f(z))| \leq M\left(\varphi, S_{0}\right)$ if $|f(z)| \leq R_{0}$ or $|z| \geq r_{0}$. These two last inequalities together imply that $\varphi \circ f \in H_{v_{c}}^{\infty}$.

To prove (2) we assume that $S_{\varphi}$ maps $H_{u}^{\infty}$ into $H_{v}^{\infty}, v(z)=\exp \left(-\frac{1}{(1-|z|)^{\beta}}\right), \beta>0$, but that there is $c>1$ such that the conclusion does not hold. The function $f(z)=\exp \left((1-z)^{-\alpha}\right)$ belongs to $H_{u}^{\infty}$ and $\|f\|_{u} \leq 1$. By assumption $\varphi \circ f \in H_{v}^{\infty}$, hence there is $M>0$ such that

$$
\log |\varphi(f(z))| \leq \log M+1 /(1-|z|)^{\beta}, \quad z \in \mathbb{D} .
$$

Select $d>1$ so that $d^{2 \beta}<c$. Since we have assumed that the conclusion of $(2)$ does not hold for $c$, we can find a sequence $\left\{w_{n}\right\}_{n}$ of complex numbers $w_{n}$ of large modulus on which the conclusion fails. Proceeding as before, we may assume that all of these points are contained in the region

$$
\Omega=\{w:|w|>\rho,-\pi / 4<\operatorname{Arg} w<\pi / 4\},
$$

for some fixed positive $\rho$. By taking a small enough positive value of $\gamma$, by considering the mapping properties of the function $z \mapsto(1-z)^{-\alpha}$, it is easy to see that every $w \in \Omega$ has a preimage in the Stolz angle of aperture $\gamma$ and vertex at $z=1$. This means that we can find a sequence of points $\left\{z_{n}\right\}_{n}$ in this angle such that $w_{n}=f\left(z_{n}\right), n \in \mathbb{N}$. Moreover, by passing to a subsequence if necessary, we can pick the points $z_{n}$ that satisfy the inequalities

$$
\left|1-z_{n}\right| \leq d\left(1-\left|z_{n}\right|\right), \quad \operatorname{Re}\left(1 /\left(1-z_{n}\right)^{\alpha}\right) \geq 1 /\left(d\left|1-z_{n}\right|\right)^{\alpha}
$$

(which simply means requiring that the points $z_{n}$ belong to the smaller of these two Stolz angles), as well as the condition $\log \left|\varphi\left(w_{n}\right)\right| \geq \log n+c\left(\log \left|w_{n}\right|\right)^{\beta / \alpha}$ for all $n \in \mathbb{N}$. Now,

$$
\left|w_{n}\right|=\exp \left(\operatorname{Re}\left(1 /\left(1-z_{n}\right)^{\alpha}\right) \geq \exp \left(1 /\left(d\left|1-z_{n}\right|\right)^{\alpha}\right) \geq \exp \left(1 /\left(d^{2}\left(1-\left|z_{n}\right|\right)\right)^{\alpha}\right),\right.
$$

for each $n \in \mathbb{N}$. This implies, for all $n \in \mathbb{N}$,

$$
\log M+\frac{1}{\left(1-\left|z_{n}\right|\right)^{\beta}} \geq \log \left|\varphi\left(w_{n}\right)\right| \geq \log n+c\left(\log \left|w_{n}\right|\right)^{\beta / \alpha} \geq \log n+\frac{c}{d^{2 \alpha}} \frac{1}{\left(1-\left|z_{n}\right|\right)^{\beta}} .
$$

This contradictions the fact that $c / d^{2 \alpha}>1$.

Of course, if $\gamma>1$, there exist entire transcendental functions $\varphi$ satisfying the assumptions of Proposition 3.6. Since these constructions are not the main subject of this article, we refer an interested reader to Corollary 1.4, Lemma 1.3 and Definition 1.1 in Langenbruch [20] or to [21].

Acknowledgements. The research of Bonet was partially supported by MICINN and FEDER Project MTM2010-15200, by GV project Prometeo/2008/101, and by ACOMP/2012/090. The research of Vukotić was partially supported by MICINN grant MTM2009-14694-C02-01, Spain and by the European ESF Network HCAA ("Harmonic and Complex Analysis and Its Applications"). 


\section{References}

[1] V. Álvarez, M.A. Márquez, D. Vukotić, Superposition operators between the Bloch space and Bergman spaces, Ark. Mat. 42 (2004), 205-216.

[2] J. Appell, P.P. Zabrejko, Nonlinear Superposition Operators, Cambridge Tracts in Mathematics 95, Cambridge University Press, 1990.

[3] J. Appell, P.P. Zabrejko, Remarks on the superposition operator problem in various function spaces, Complex Var. Elliptic Equ. 55 (2010), no. 8-10, 727-737.

[4] K.D. Bierstedt, J. Bonet, A. Galbis, Weighted spaces of holomorphic functions on bounded domains, Michigan Math. J. 40 (1993), 271-297.

[5] K.D. Bierstedt, J. Bonet, J. Taskinen, Associated weights and spaces of holomorphic functions, Studia Math. 127 (1998), 137-168.

[6] J. Bonet, P. Domański, M. Lindström, Essential norm and weak compactness of composition operators on weighted Banach spaces of analytic functions, Canad. Math. Bull. 42, no. 2, (1999), 139-148.

[7] J. Bonet, P. Domański, M. Lindström, J. Taskinen, Composition operators between weighted Banach spaces of analytic functions, J. Austral. Math. Soc. (Series A) 64 (1998), 101-118.

[8] C. Boyd, P. Rueda, Holomorphic superposition operators between Banach function spaces, Preprint, 2011.

[9] C. Boyd, P. Rueda, Superposition operators between weighted spaces of analytic functions, Preprint, 2011.

[10] S.M. Buckley, J.L. Fernández, D. Vukotić, Superposition operators on Dirichlet type spaces, in Papers on Analysis: A Volume dedicaed to Olli Martio on the occasion of his 60th birthday, pp. 41-61, Rep. Univ. Jyväskyla Dept. Math. Stat. 83, Univ. Jyväskyla, Jyväskyla, 2001.

[11] S.M. Buckley, D. Vukotić, Univalent interpolation in Besov spaces and superposition into Bergman spaces, Potential Anal. 29 (2008), No. 1, 1-16.

[12] G.A. Cámera, Nonlinear superposition on spaces of analytic functions, in Harmonic Analysis and Operator Theory (Carácas, 1994), Contemp. Math. 189, pp. 103-116, Amer. Math. Soc. Providence RI, 1995.

[13] G.A. Cámera, J. Giménez, The nonlinear superposition operators acting on Bergman spaces, Compositio Math. 93 (1994), 23-35.

[14] S. Dineen, Complex Analysis in Locally Convex Spaces, North-Holland Math. Studies 57, Amsterdam 1981.

[15] J.J. Donaire, D. Girela, D. Vukotić, On univalent functions in some Möbius invariant spaces, J. reine angew. Math. 553 (2002), 43-72.

[16] R.E. Castillo, J.C. Ramos Fernández, M. Salazar, Bounded superposition operators between Bloch-Orlicz and $\alpha$-Bloch spaces, Appl. Math. Comp. 218 (2011), 3441-3450

[17] D. Girela, M.A. Márquez, Superposition operators between $Q_{p}$ spaces and Hardy spaces, $J$. Math. Anal. Appl. 364 (2010), 463-472.

[18] K.-G. Grosse-Erdmann, A weak criterion for vector-valued holomorphic functions, Math. Proc. Camb. Publ. Soc. 136 (2004), 399-411.

[19] A. Harutyunyan, W. Lusky, On the boundedness of the differentiation operator between weighted spaces of holomorphic functions, Studia Math. 184 (2008), 233247. 
[20] M. Langenbruch, Continuation of Gevrey regularity for solutions of partial differential operators, pp. 249-280 in: Functional Analysis (Trier, 1994), de Gruyter, Berlin, 1996.

[21] B.Ya. Levin, Lectures on Entire Functions, Translations of Mathematical Monographs 150, Amer. Math. Soc., Providence, RI, 1996.

[22] W. Lusky, On weighted spaces of harmonic and holomorphic functions, J. London Math. Soc. 51 (1995), 309-320.

[23] W. Lusky, On the isomorphism classes of weighted spaces of harmonic and holomorphic functions. Studia Math. 175 (2006), 19-45.

[24] Ch. Pommerenke, Boundary Behaviour of Conformal Maps, Springer-Verlag, Berlin, 1992.

[25] J.C. Ramos Fernández, Bounded superposition operators between weighted Banach spaces of analytic functions, preprint, available from http://arxiv.org/abs/1203.5857.

[26] A.L. Shields, D.L. Williams, Bounded projections, duality and multipliers in spaces of analytic functions, Trans. Amer. Math. Soc. 162 (1971), 287-302.

[27] D. Vukotić, Integrability, growth of conformal maps, and superposition operators, Technical Report 10, Department of Mathematics, Aristotle University of Thessaloniki, 2004.

[28] C. Xiong, Superposition operators between $Q_{p}$ spaces and Bloch-type spaces, Complex Var. Theory Appl. 50 (2005), 935-938.

[29] W. Xu, Superposition operators on Bloch-type spaces, Comput. Methods Funct. Theory 7 (2007), 501-507.

[30] K. Zhu, Operator Theory in Function Spaces. Second Edition, Amer. Math. Soc., 2007. 